\title{
DUALITY AND SCALING IN QUANTUM MECHANICS
}

Dhurjati Prasad Datta

Department of Mathematics

North Eastern Regional Institute of Science And Technology

Itanagar-791109,Arunachal Pradesh, INDIA*

and

Inter-University Centre for Astronomy and Astrophysics

Ganeshkhind, Pune-411007, INDIA

email:dpd@nerist.ernet.in

\begin{abstract}
The nonadiabatic geometric phase in a time dependent quantum evolution is shown to provide an intrinsic concept of time having dual properties relative to the external time.A nontrivial extension of the ordinary quantum mechanics is thus obtained with interesting scaling laws.A fractal like structure in time is thus revealed. PACS nos.: 03.65.Bz.; 04.60.-m; 47.53.+n

Keywords:Geometric phase, Internal time, Duality, Scaling, Fractal
\end{abstract}

*Permanent address 
A generic property of a time dependent quantal evolution is that the quantal system picks a nonzero geometric phase[1], besides the ordinary dynamical phase indicating the flow of time.The existence of a geometric phase in turn indicates the presence of a small scale motion in the system[2,3], whose origin could be traced to the geometry(curvature) of the projective Hilbert space. The physical implications of this quantal geometric motion are being investigated in a series of papers recently[3].The motivation of these studies comes mainly from quantum gravity(cosmology).A well known problem in the canonical quantum gravity is the absence of an a priori external concept of time at the quantum level.The possible emergence of time intrinsically from the dynamics of an interacting system is of much relevance here.Indeed it is shown[3],in particular, that an internal concept of time could be realized in an interacting system in connection with the nonadiabatic geometric phase picked by a pertinent quantal state.Although derived in the framework of a quantum cosmological Wheeler-Dewitt (WD) equation,this and other related results, however, turn out to be more universal in character.It is therefore of much interest to investigate analogous results in ordinary quantum mechanics.In this letter,we do precisely this by reporting some preliminary,but novel results in this direction.

The mean energy in a quantal state defines a scale of the external (Newtonian) time, which in turn is assumed usually to parametrize the path of the evolving state in the relevant Hilbert space. The actual evolution of the quantal state however consists of two independent components: the dominant, purely dynamical, evolution in connection with the mean energy of the state, accompanied with a small geometric component appearing as fluctuations on the mean evolution. Treating the Hilbert space as a U(1) principal bundle over the projective space of rays, the mean evolution could in turn be represented as a pure vertical motion along the fibre corresponding to the state.This dominant vertical component in the quantal evolution thus induces a change of phase in the state:the so called dynamical phase.The (nonadiabatic) geometric phase, on the otherhand, is a consequence of the small scale geometric motion in the state. The inherent geometric nature of this phase is captured by the associated parallel transport law which not only states how the state is being parallely transported along a horizontal curve in the projective space but also provides a natural framework for its actual computation[1,2].However, there is an equivalent derivation[4] which treats the geometric phase instead as a dynamical one, thus realizing it as a correction term in the total phase.In the following we shall make use of this later approach in our discussion of the internal time.

As remarked already,the nonadiabatic geometric phase is related to the quantal fluctuations in the state in a natural way.Consequently,the geometric contribution in the vertical motion in the state is, in fact, due to these irreducible fluctuations.In fact, we shall show that an intrinsic description of the quantal evolution could be made meaningful in association with the nonadi- 
abatic geometric phase and hence the fluctuations in the state. The small vertical displacement of the state due to the nonadiabatic phase could indeed be interpreted locally (in the neighbourhood of a given instant of the external time) as a (dominant) dynamical evolution in the relatively small scale of the internal time variable,having conjugate relationship with the root mean square energy fluctuation (uncertainty).The term 'internal' qualifies the fact that it is an intrinsic gauge invariant geometrical object constructed out of the quantal state itself (eg.,the Fubini-Study metric in the projective space) without any reference to an external concept[5].In quantum cosmology, this internal time variable, after a proper rescaling,could be identified by an internal observer as the observable time in the universe,reasonably well-defined for the description of the local physics[3].Note that the fluctuations in the original state not only will appear relatively large $\mathrm{O}(1)$ with respect to this small scale internal time, but an (internal) observer equipped with this internal time and the associated rescaled Schrodinger equation (cf.,eq.(11)) even identifies a 'large' (external) fluctuation as the mean energy in the pertinent internal state.Because of the irreducibility of the quantal fluctuations, the above process could be iterated ad infinitum, thus establishing self-similar fluctuations at all (time) scales in a non-stationary quantal evolution.

Using the technique of the renormalization group (RG) as in the statistical mechanical models near a critical point,we explore these self-similar quantal fluctuations in the extended framework of an internal time.The RG critical point corresponds here to the short time (large fluctuation) limit of the quantal evolution.In that limit the external and internal time variables are found to be dually related,reminiscent of the recently studied string dualities[6].Further, the nontrivial scaling of the two point time-time correlator is interpreted as a signature of the fractality of time. We also explore some physical (experimental) implications of the fractal time.

Let us consider a quantal evolution of a state $\mid \psi(t)>$ given by the Schrodinger equation $(\hbar=1)$

$$
i \frac{d}{d t}|\psi>=H| \psi>
$$

where $H$ denotes the Hermitian Hamiltonian operator and $t$ is the external time. For definiteness, we assume that the potential energy function is bounded from below.Let $H$ denote the Hilbert space of the normalized states: $\langle\psi \mid \psi\rangle=$ 1. Then $H$ is the $\mathrm{U}(1)$ principal bundle on the projective space $\mathcal{P}$ of rays. Recall[7] that a nonstationary isolated state moves around $\mathcal{P}$ provided the cor-

1 The adiabatic phase,on the contrary, is nonzero only for a parameter space with nontrivial geometry( topology).Although an internal concept of time could even be attached to a nontrivial adiabatic phase,this would only be a special feature of the specific parameter space chosen to describe the quantal adiabatic motion. The internal time in connection with a nonadiabatic phase, on the other hand, is uniquely defined by the Fubini-study metric in the projective space and hence has a universal character(see the main text for details. 
responding uncertainty $\Delta E$ in the energy: $\Delta E=\sqrt{\left\langle\psi\left|(H-E)^{2}\right| \psi\right\rangle}, E=<$ $\psi \mid H \psi>$,is nonzero. Further, the characteristic time scale of the dynamics is fixed by $t \approx 1 / E$ with the auxiliary condition that $\Delta E \rightarrow 0$ in the adiabatic limit $t \rightarrow t_{0}$, which we assume as the initial condition. We restrict the discussion to the evolution close to $t \rightarrow t_{0}$, when the nonadiabatic corrections become important. More general initial conditions will be treated elsewhere.

In the presence of a geometric phase (nonzero for a nonstationary state) the time derivative in the Schrodinger equation (1) can be interpreted as a total derivative

$$
\frac{D}{d t}=\frac{\partial}{\partial t}+\frac{d}{d t}=\frac{\partial}{\partial t}+\frac{d q_{i}}{d t} \frac{\partial}{\partial q_{i}}
$$

The first term in the right hand side takes care of the explicit time dependence, whereas the second term indicates the implicit time dependence through some time dependent parameters $q$. The parameters $q$ can even be identified as the co-ordinates of the projective space $\mathcal{P}[4,7]$. The adiabatic parameters of Berry[1] belongs,in particular,to a subclass of this general set of parameters.In the following we use the same notation $\mathcal{P}$ to denote either of these two spaces, the projective or the general parameter space, which one is appropriate. Nevertheless, our discussion is independent of any specific choice of the time dependent parameter space, the nonadiabatic geometric phase being solely an intrinsic property of the projective space.

Projecting out the the dynamical phase of the state $|\psi\rangle$, eq.(1) reduces to

$$
i \frac{D}{d t}|\phi>=\tilde{H}| \phi>, \quad \tilde{H}=H-E
$$

where $\left|\phi>=e^{i \int E d t}\right| \psi>$. The state $\mid \phi>$ now belongs to the horizontal subspace[1] of the tangent space of $H$ at the point $|\psi\rangle$,defined via the parallel transport law

$$
<\phi\left|\frac{D}{d t}\right| \phi>=0
$$

The geometric phase $\gamma$ for a closed path $C$ in $\mathcal{P}$ is now given by the holonomy integral for the connection 1-form $A$

$$
A=-<\tilde{\phi} \mid i d \tilde{\phi}>, \quad \phi=e^{i \gamma} \tilde{\phi}, \quad \tilde{\phi} \in \mathcal{P}
$$

$d$ being the exterior derivative in $\mathcal{P}$. For an open path the corresponding phase (the Pancharatnam phase) is given by an integral along the shortest geodesic joining the initial and the final rays[8].

Alternatively, eq.(3) offers itself to a nonstandard interpretation[3]: one could, in fact, define an intrinsic concept of time in the dynamics using the nontriviality of the connection form (5). To begin with,recall that there is 
an alternative derivation which realizes the anholonomic phase $\gamma$ instead as a dynamical phase[4].Introduce a unitary transformation on the state $\mid \phi>$

$$
|\phi>=U| \chi>
$$

so that the transformed state $\mid \chi>$ may only have an implicit time dependence: $\frac{\partial}{\partial t} \mid \chi>=0$. Eq.(3) becomes

$$
i \frac{d}{d t}\left|\chi>=h_{0}\right| \chi>
$$

where $h_{0}=U^{\dagger} \tilde{H} U-i U^{\dagger} \dot{U}$, and $\dot{U}=\frac{\partial U}{\partial t}$. We note that such a unitary transformation always exists[4](see Appendix for a proof).Eqns.(6)-(7) and (4) now give $i<\chi|i d \chi>=<\chi| h_{0}|\chi>d t=-i \chi| U^{\dagger} \partial U|\chi>=-i<\phi| \partial \phi>=A$, when one makes use of $\partial U \mid \chi>=\partial(U \mid \chi>)$. Thus the phase $\gamma$ is realized as a dynamical phase for eq.(7).Note that the parameter $t$ in the implicit time derivative in the lhs of eq. (7) parametrizes the path traced by the quantal state as an integral curve of the vector field $(d / d t)=\left(d q_{i} / d t\right)\left(\partial / \partial q_{i}\right)$ (cf.eq.(2)) in $\mathcal{P}$ and acts as a dummy variable. Note also the reparametrization invariance of the equality

$$
<\chi|i d \chi>=-i<\chi| U^{\dagger} \partial U \mid \chi>
$$

which lies at the heart of our discussion of the internal time.

The philosophy of the internal time, advocated originally by Leibniz, was discussed at lenght by Barbour[5] recently. This intrinsic Leibniz concept of time (and also space) satisfies the Mach-Einstein relativity principle. As pointed out by Barbour a gauge invariant formalism of the dynamics using intrinsic (relative) concepts ought to yield nontrivial predictions. The inherent reparametrization invariance in the intrinsic description, for instance, results in a nontrivial Hamiltonian constraint even in the ordinary (nongravitational) dynamics. The internal time introduced on the basis of eq.(8) (and eq.(7)) (see below) clearly is a realization of the so called Leibniz time.This offers a most economical description of the quantal evolution of the fluctuating state over the instantaneous state $|\phi\rangle$, without necessitating a reference to the external Newtonian time $t$.For, the constraint alluded to above corresponds, in the present discussion, to $\langle\phi|H-E| \phi\rangle=0$, which in the adiabatic limit leads to the generalized WD equation[3]

$$
\tilde{H}|\phi>\equiv(H-E)| \phi>=0
$$

for a fixed $E$.Recall that the intrinsic geometric motion in the instantaneous eigenstate $\mid \phi>$ is encoded in the parallel transport law (4).Eq.(8), on the otherhand, treats this geometric motion on a 'dynamical' footing by transferring to a quantal moving frame eq.(6) attached to $\mid \phi>$. The phase $\gamma$ now corresponds 
to the correction (due to quantal fluctuations) $\Delta E$ over the energy $E$,provided the operator $U$ is identified with the interaction picture evolution operator:

$$
i \frac{\partial}{\partial \tau} U=\tilde{H} U(\tau), \quad \tilde{H}(\tau)=H(\tau)-H_{0}
$$

where $H_{0}=H\left(t_{0}\right)=H(\tau=0)$ and $U(0)=I$. Following the Leibniz view[5], the internal time $\tau$ must truly be an intrinsic variable, which relates $d \tau$ uniquely to the Fubini-Study arclength giving the distance between two infinitesimally separated states in the projective space $\mathcal{P}[3]$. This follows from the fact that the (dimensionless) Fubini-Study arclength $d s$ (say) relates to the actual motion of the nonstationary quantal state $|\psi\rangle$ in the projective space $\mathcal{P}$ through the gauge invariant relation $d s=2 \Delta E d \tau$ [7].'] The internal time $\tau$ is thus uniquely defined and is independent of the choice of the parameters $q$.The distinguished intrinsic variable $(\tau)$ thus makes explicit the dynamical content of the parallel transport law (4) by breaking its reparametrization invariance dynamically.

The intrinsic Schrodinger equation which follows the residual geometric motion of the instantaneous eigenstate $\mid \phi>$ is thus obtained as

$$
i \frac{d}{d \tau}|\chi(\tau)>=-h(\tau)| \chi(\tau)>
$$

where $h(\tau)=U^{\dagger}(\tau) \tilde{H}(\tau) U(\tau)$. Note also that $<\chi|h| \chi>=\sqrt{\left\langle\psi\left|\tilde{H}^{2}\right| \psi\right\rangle}$, by construction. Eqs.(10) and (11) are the exact analogs of an equivalent set of equations viz.,eqs.(8) and (9) in Ref.[3,the CQG paper] for the heavy (gravitational) degrees of freedom and the lighter matter states respectively,which were derived from a quantum cosmological WD equation by using the semiclassical Born-Oppenheimer approximation.The present treatment, on the otherhand, is fully quantal and of more general nature.It is gratifying that the internal time introduced here turns out to be consistent with the relevant concept in quantum gravity(cosmology).An 'internal observer' belonging to the 'WD state' $\mid \phi>$ will then identify the state $\mid \chi(\tau)>$ as a vertical state indicating the (small scale) evolution in time $\tau$. The internal state $\mid \chi(\tau)>$ and the external horizontal state $\mid \phi(t)>$ (cf.eq.(4)) are,however, related by a gauge rotation.We remark that eq.(11) offers novel physical predictions.In fact,in an intrinsic description based on Eq.(11) one not only identifies the uncertainty $\Delta E$ in the state $\mid \psi>$ (eq.(1)) as the energy for the (internal) state $\mid \chi>$, but

\footnotetext{
${ }^{2}$ In ref.[7] the relation $d s=2 \Delta E d t$ is obtained in the ordinary quantum mechanics. However, $d s$ is a property of the unparametrized curve in $\mathcal{P}$ and hence the parameter $t$ acts as a dummy variable. In the present intrinsic description the dummy variable $\tau$ is raised to the status of the (internal) time via the definition $d \tau=d s / 2 \Delta E$.

${ }^{3}$ It follows from eqs.(2)-(5) and (11) that $-i U \frac{d}{d \tau} U^{\dagger}=i \frac{D}{d t}=i \frac{\partial}{\partial t}+\tilde{H}+\frac{\tilde{H}^{2}}{\Delta E}$. The implicit time derivative in eq.(1) is thus related to energy uncertainty, in contrast to the concomitance of the explicit derivative and energy.
} 
the original energy $E$ of $\mid \psi>$ turns out also to be unobservable[3]. This should be contrasted with the relative smallness of $\Delta E$ (actually unobservable, in the adiabatic limit) in the extrinsic description,eq.(1).

What if one makes use of the original external time $t$ in eqns.(10)-(11) instead of $\tau$ ?Clearly,in this extrinsic description time $t$ scales as $E^{-1}$. The fluctuations in the state would therefore be small,at least in the adiabatic $\operatorname{limit}\left(t \rightarrow t_{0}\right)$. Then $\Delta E \rightarrow 0$ as $\tilde{t} \rightarrow 0, \tilde{t}=t-t_{0}$. Eq.(11),on the otherhand, indicates that $\Delta E$ scales as $-\left(t-t_{0}\right)^{-1}$. This dichotomy can be resolved only if one sets the initial time uniquely at $t_{0}=-\infty$. This choice of initial condition, though appears reasonable for an isolated scattering state, is nevertheless too restrictive for strongly interacting systems. The present intrinsic description should therefore be of interest not only in quantum gravity but also in posing the initial value problem for a general (strongly) interacting quantum system.

Note that the (-)sign in eq.(11) takes care of the fact that the direction of traversal of a path in $\mathcal{P}$ as seen externally is reversed internally.Further,as noted above the internal time scales as $(\Delta E)^{-1}$, in contrast to the external time behaviour: $\Delta E \rightarrow 0$ as $\tilde{t} \rightarrow 0$. Moreover, in view of the relation $t \approx E^{-1}$; $\tilde{t} \approx E^{-2} \delta E$ and thus the $\tilde{t} \rightarrow 0$ limit is reached either as $\delta E \rightarrow 0$ (the external stationarity) or by taking $E \rightarrow \infty$ (asymptotic high energy region). Further,in relation to a very small time scale (when the concept of the internal time becomes relevant) both the energy differential $\delta E$ and the uncertainty $\Delta E$ will appear large and comparable, $\approx O(E)$. In that limit of large fluctuations time variables $\tilde{t}$ and $\tau$ are related by the duality relation $\tilde{t}=1 / \tau$. Here, we measure both the time variables in the unit of $E$. In the following we omit tilde and assume $t \rightarrow 0$. We next show by following the RG technique that the behaviour of a quantal evolution may undergo a change at the self-dual point $t=\tau(=1)$, analogous to a phase transition at a critical point.However, before delving into this discussion we first give an example illustrating briefly some of the results discussed so far.

Let us consider a time dependent harmonic oscillator given by the Hamiltonian [9], $H(t)=\left(p^{2} / 2 m\right)+m w^{2}(t) q^{2} / 2$. The time dependent state $\mid \chi>$ can be determined by the $\mathrm{so}(2,1)$ valued invariant operator method[for details we refer to [9]]. The mean energy in the state $|\psi\rangle$, in the short time limit $t \rightarrow 0$ near an initial adiabatic state $\mid \psi(0)>$ can be written as $E=E_{0}(1+\nu)$, where $E_{0}=w_{0}(n+1 / 2), w_{0}=w(0), \nu=2|\beta|^{2}, \beta=$ the Bogoliubov $\beta$-coefficient. Then $\Delta E=\nu E_{0} \approx$ the uncertainty in $\mid \psi>$ when $\nu \approx O(1)$. Further the nonadiabatic phase can be computed $[10,3]$ as $\gamma=\Delta E t$. Note that the total phase remains equal to $\left(E_{0} t\right)$, in the present approximation. Clearly as the external time scales as $t \approx \nu E, \nu \rightarrow 0 ; \Delta E t \approx \nu^{2} \rightarrow 0$. However, $\gamma$ can be freezed in the time variable $\tau \approx(\nu E)^{-1}$, letting $\Delta E \tau \approx 1$. In this intrinsic description $\tau \rightarrow 0 \Rightarrow \nu \rightarrow \infty$. Interestingly, the scaled Hamiltonian in Eq.(11) can be obtained as $h(\tau)=\nu H(\tau)$. Further details, however, will be reported in ref.[10].We conclude this illustration with a remark. The adiabatic initial condi- 
tion is assumed here for the sake of computational ease.In principle one could as well start with a strongly interacting initial state, which would then lead to an adiabatic state in the dual intrinsic description.

To return to the main body of the analysis, let us recall that our intention is to study the nature of the quantal evolution near the self dual point $t=\tau$. To this effect,let $\psi_{R G}(0, t)$ denote the two point time-time correlation function of the (Euclidean) Schrodinger equation. Then $\psi_{R G}=<\psi(0) \mid \psi(t)>$, where $\mid \psi>$ denotes the purely time dependent factor of the Euclidean Schrodinger wave function henceforth. One may interpret it as the transition probability of the state from the initial time $t=0$ to the final time $t$, with energy $E$. In the conventional extrinsic treatment of quantum mechanics, the probability is 1 , in the adiabatic limit $t \rightarrow 0$. In reality, however, fluctuations are nonzero, making room for an intrinsic description as well. A given state may thus have two possible paths, given by either of the two equations viz.,eq.(1) or (11), for its evolution near $t=0(\tau=0)$. Consequently,as we now show, the probability ceases to be unity.

Writing $\frac{d \gamma}{d t}=\nu E$, and introducing the Wick rotation $t \rightarrow-i t$, eq.(1) (together with (2)) is expressed in the form of a Callan-Symanzik RG equation[11]

$$
\left(\frac{1}{E} \frac{\partial}{\partial t}+\beta(\tau) \frac{\partial}{\partial \tau}+1+\nu\right) \psi_{R G}=0
$$

where the $\beta$-function is given by $\beta(\tau)=\frac{d \tau}{d t}$, and $E d \tau \rightarrow d \tau$. Eq.(12) is obtained by projecting the Wick rotated equation (1) on the initial state $|\psi(0)\rangle$, since $\frac{D}{d t}<\psi(0)|\psi(t)>=<\psi(0)| \frac{D}{d t} \psi(t)>$. The homogeneous equation (12) restricts $t>0$. Note that in the extrinsic description, $\tau \equiv t$; leading to the trivial scaling (see below). We are, however, interested in the nontrivial case when $\tau$ is the internal time. The $\beta$-function is then computed by recalling that $t$ and $\tau$ are duality variables: $\beta(\tau)=-\tau^{2} . \quad \tau=0$ is thus a(n) (ultraviolate stable) fixed point of the $\beta$-function. This could be interpreted as a sort of an 'asymptotic freedom' in the nonstationary dynamical system. In fact the nontrivial 'asymptotic freedom' is a manifestation of a competition between two different limits $t \rightarrow 0$ (externally) and $\tau \rightarrow \infty$ (internally) near the fixed point. Consequently, a strongly interacting system could be mapped to a weakly interacting one by the duality transformation. Another important effect of this duality is the stabilization of the 'anomalous dimension' $\nu$ to a nonzero finite value. This is obtained by comparing the two Schrodinger equations (1) and (11), which should agree at the fixed point. One obtains $(1+\nu) E d t=$ $-\nu E d \tau \Rightarrow 1+\nu=\nu \tau^{2}$, which in the limit $t \rightarrow 0, \tau \approx \nu^{-1} \rightarrow \infty$; gives the fixed point equation $1+\nu_{0}=\nu_{0}^{-1}$, yielding the limiting value of the 'anomalous dimension' $\nu$ as the golden mean $\nu_{0}=\frac{1}{2}(\sqrt{5}-1)$. The nonzero value of $\nu$ in the present case should be contrasted with the vanishing of the same in the case of a gauge theory asymptotic freedom. The final form of the solution of the RG equation (12) can now be written as $\psi_{R G}=\sigma^{-1-\nu} g(\tau)$, where $g$ 
is an arbitrary function of the running 'coupling constant' $\tilde{\tau}$ satisfying the boundary condition $g=$ const., at $\tilde{\tau}=0$.. Here $\sigma$ is an energy scaling variable. One thus obtains the nontrivial scaling for the two point correlation function in the asymptotic region : $\psi_{R G} \approx \sigma^{-1-\nu_{0}}$. For the sake of comparison, we note that the expected scaling in the extrinsic description, which can be directly verified is $\psi_{R G}^{0} \approx \sigma^{-1}$. To get better insights into the scaling law, we take the inverse Laplace transform(as we consider $t>0$ ) of the same so as to express it in the time coordinate: $\psi_{R G} \approx t^{\nu_{0}}\left(\psi_{R G}^{0} \approx 1\right)$.

Clearly, a natural interpretation of this scaling behaviour could be obtained in the framework of the fractal geometry. The scaling law tells us that the fixed point $t=0$ is an extended object with finer fractal-like structures. By the time translation invariance of the Schrodinger equation it follows that every point in the time axis has identical fractal structure. The extended time axis is thus a fat fractal [12], with the uncertainty exponent $\nu_{0}$. An interesting physical implication of this uncertainty exponent is the following: Near the fixed point both the Schrodinger equations (1) and (11) could, in principle, be considered as the correct evolution equations with definite but distinct physical predictions for a given system.Moreover,these distinct physical possibilities of a given state,are in a state of an inextricable mixture in view of the fractal nature of the fixed point.An experiment intending to measure the (geometric) phase (for a very short time evolution) of the state should, therefore, reveal a randomness in the observed values. One expects to see, for instance, a data consisting of a random distribution of two numbers,e.g.,1(suitably normalized value of the phase on the basis of eq.(1)) and 0 (for eq.(11)),provided repeated measurements are made, on identically prepared states, for a considerable period of time.A very high resolution (phase determination) experiment on a nonadiabatic quantal system in the region of (relatively) large fluctuations may perhaps confirm the prediction.If so,this may be considered as an experimental test for the fractal nature of time, which might have important physical implications[3]. We conclude by noting that by inhabiting a duality transformation,the fractal time extends the linear time translation invariance of the Schrodinger equation to an action of the $\mathrm{SL}(2, \mathrm{R})$ transformations.Further details of this group action in quantum mechanics and the other related issues will be considered separately.

\section{Acknowledgements}

It is a pleasure to thank T.Padmanabhan and S.Sinha for discussions and Inter University centre for Astronomy and Astrophysics,Pune for awarding a Senior Associateship. The work is also supported by the Department of Science and Technology, Government of India. 


\section{References}

[1] A.Shapere and F. Wilczek(eds.), Geometric phases in Physics (World Scientific, Singapore, 1989).

[2] J.Anandan, Nature 360,(1992) 307.

[3] D.P.Datta,Class.Quant.Grav.12,(1995)2499;Phys.Rev.D50,(1994) 2605 ; ibid52,(1995) 3375;Int.J.Mod.Phys.D 5 (1996) 433.

[4] M.V.Berry,The quantum phase, five years after, in: ref.[1]; Proc. R. Soc. Lond. A 414,(1987) 31; L.A.Wu, J.Sun, and J.-Y. Zhong, Phys. Lett. A, 183, (1993) 257.

[5] J.B.Barbour, in Quantum concepts in space and time, eds: R. Penrose and C.J.Isham (Oxford University Press,Oxford,1986).

[6] E. Alvarez, L. Alvarez-Gaume and Y. Lozano, Nucl. Phys. B(Proc. suppl.) 41, (1995) 1.

[7] J. Anandan and Y. Aharonov, Phys. Rev. Lett.65,(1990) 1697 ; J. Anandan, in Quantum Coherence, ed. J. Anandan, World Scientific (1990).

[8] J. Anandan and Y. Aharonov, Phys. Rev. D 38, (1988) 1863.

[9] S.P.Kim, J. Phys. A: Math. Gen. 27,(1994) 3927.

[10] D.P.Datta, in preparation.

[11] T.-P.Cheng and L.-F.Li, Gauge theory of elementary particles, (Oxford University Press, Oxford,1984).

[12] E.Ott,Chaos in dynamical systems (Cambridge University Press, Cambridge, 1993).

\section{APPENDIX}

Theorem:Given the Schrodinger equations (3) and (7),there exists a unitary transformation $U$ (Eq.(6)) between them provided $(H-h)$ is not implicitly time dependent .

The proof follows from an explicit construction. Let $U=U_{0}\left(t, t_{0}\right) U_{1}^{\dagger}\left(t, t_{0}\right)$, where $U_{0}$ and $U_{1}$ be the evolution operators of Eqs.(3) and (7) respectively. Then $U$ satisfies the relation $h_{0}=U^{\dagger} \tilde{H} U-i U^{\dagger} \dot{U}$, provided $h_{0}=i U_{0}^{\dagger} \frac{d}{d t} U_{0}$. The theorem follows once we split $U_{0}$ further into an explicit and an implicit time dependent unitary opera- tors: $U_{0}=U_{e x} U_{i m}$. This splitting is certainly possible for the class of models with $H=H_{e x}+H_{i m},\left[H_{e x}, H_{i m}\right]=0$. In the present discussion $H_{e x}=0$. 